\title{
Anti-Leukemic Properties of Bacterial L-Asparaginase
}

\author{
Yago Queiroz dos Santos ${ }^{1,4 *}$, Gabriella Silva Campos Carelli ${ }^{2,3}$, Bruno Oliveira de Veras $^{3}$ and \\ Elizeu Antunes dos Santos ${ }^{2}$ \\ ${ }^{1}$ Federal Institute of Education, Science and Technology of Ceará, Brazil \\ ${ }^{2}$ Department of Biochemistry, Brazil \\ ${ }^{3}$ Institute of Tropical Medicine, Brazil \\ *Corresponding author: Yago Queiroz dos Santos, Federal Institute of Education, Science and Technology of Ceará, Boa Viagem, \\ Ceará, Institute of Tropical Medicine, Federal University of Rio Grande do Norte, Natal, Rio Grande do Norte, Brazil
}

\section{ARTICLE INFO}

Received: 㓞 September 05, 2019

Published: 靟 September 12, 2019

Citation: Yago Queiroz dos S, Gabriella Silva Campos C, Bruno Oliveira de V, Elizeu Antunes dos S. Anti-Leukemic Properties of Bacterial L-Asparaginase. Biomed J Sci \& Tech Res 21(2)-2019. BJSTR. MS.ID.003584.

Keywords: L-Asparaginase; Leukemia; Bacteria; ALL

\begin{abstract}
L-asparaginase is a ubiquitously found enzyme in organisms, having the ability to selectively hydrolyze the amino acid L-asparagine releasing ammonia and L-aspartate. The catalyzed L-asparaginase reaction has been explored in the clinic for the treatment of leukemia and other hematopoietic disorders, especially for the treatment of acute lymphoblastic leukemia, a cancer with high prevalence in young people up to 20 years old. Current drugs available on the market have been in use for over 30 years, and new therapeutic formulations need to be established and a detailed study of the mechanism of action of asparaginases to induce tumor cell apoptosis. Marine bacteria are a potential source for prospecting for new, more active asparaginase isoforms when compared to the enzymes already described and are potentially more stable under extreme temperature and $\mathrm{pH}$ conditions, thus allowing greater variability in their formulation and administration. The characterization of new asparaginases from sources not yet described in the literature as marine microorganisms is essential in order to evaluate the antitumor potential of this new drug in in vitro models involving leukemic and solid tumor strains, aiming at proposing a new pharmacological formulation to current therapeutic strategies available for the treatment of acute lymphoblastic leukemia.
\end{abstract}

\section{Background}

Leukemia is a pathology with an etiology not yet described, related to the disordered and irregular development of certain cell types of the hematopoietic system. It is one of the cancers with considerable prevalence in specific portions of the population and is characterized by abnormal proliferation of blast cells of the myelocytic or lymphocytic lineage in the bone marrow and peripheral blood that tend to replace normal leukocyte cells Sourani et al. [1]. Acute lymphoblastic (or lymphoid) leukemia (ALL) accounts for $12 \%$ of all leukemias diagnosed in the United States, and $60 \%$ of cases occur in people under the age of 20 . ALL is the most common cancer in cancer patients under 15 years. Lymphoid leukemias are aggressive and rapidly progressing. Due to these factors, speed and efficiency in diagnosis and treatment are required Izadpanah Qeshmi et al. [2]. L-Asparaginase is an important chemotherapeutic agent employed in the treatment of acute lymphoblastic leukemia and other hematopoietic syndromes.
In the early 1950's, Kidd and colleagues identified that the Cavia porcellus rodent serum had the ability to control the progression of a lymphoma: mice were transplanted with lymphoma cells and repeated intraperitoneal injections of normal Cavia porcellus serum were administered. This treatment led to a regression of lymphoma and increased survival of treated rats while progressive lymphoma growth and death within 20-30 days were observed in the control. Such regression ability was posteriorly confirmed, with L-asparaginase isolated from Cavia porcellus serum and capable of presenting the same results against lymphoma alone Wriston and Yellin [3]. Unlike normal cells, leukemic cells have a serious depletion in Asparagine Synthetase enzyme activity and are therefore unable to perform asparagine synthesis via the pathways again. Given the high requirement for exogenous asparagine, a deprivation for leukemic cells of this amino acid results in inhibition of protein synthesis and subsequent death of tumor cells Chen [4]. 
Because of this high demand for exogenous asparagine, tumor cells are directly affected by catalysis promoted by the enzyme L-asparaginase (EC 3.5.1.1) which is capable of selectively hydrolyzing the amino acid L-asparagine (Asn) in aspartic acid and ammonia, significantly decreasing serum Asn levels (Figure 1). During the L-asparaginase reaction, a covalent intermediate (Beta-acyl enzyme) is formed by an enzymatic nucleophilic attack. The gray arrow indicates a nucleophilic attack and the last black arrow a hydrolysis. This beta-acyl intermediate will later become a L-aspartate within the liberation of ammonia. Normal cells are protected against asparagine requirement because of their ability to produce this amino acid, so unlike conventional cancer therapy, L-asparaginase-based leukemia therapy is highly specific Shrivastava et al. [5]. Despite all the advantages of treating ALL, the currently used clinical L-Asparaginase formulations still need improvement due to the emerging resistance of tumors and still retain several adverse effects such as the high immunogenicity that these molecules still have. Thus, the characterization of new asparaginases from sources not yet described in the literature as marine microorganisms is a fundamental part for the therapeutic continuity against leukemias and other hematopoietic syndromes Zuo et al. [6].

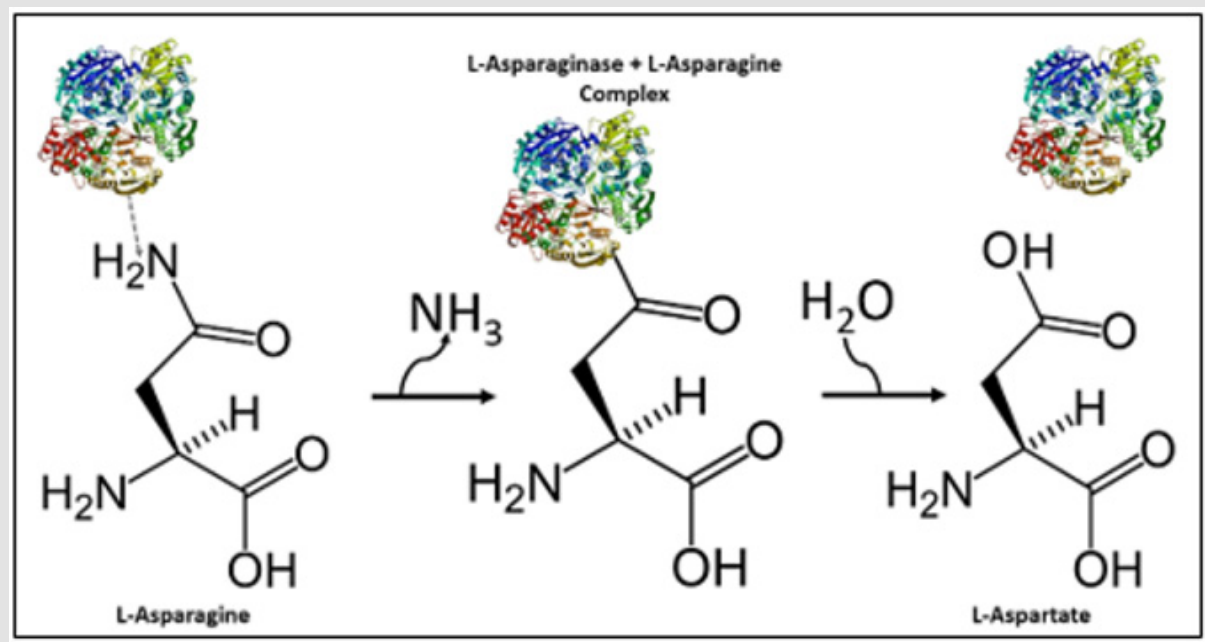

Figure 1: L-Asparaginase reaction mechanism.

\section{References}

1. Sourani Z, H Shirzad, M Shirzad, B Pourgheysari (2017) Interaction between Gallic Acid and Asparaginase to Potentiate Anti-Proliferative Effect on Lymphoblastic Leukemia Cell Line. Biomedicine and Pharmacotherapy 96: 1045-1054.

2. Izadpanah Qeshmi, Fatemeh, Ahmad Homaei, Pedro Fernandes, Sedigheh Javadpour, et al. (2018) Marine Microbial L-Asparaginase: Biochemistry, Molecular Approaches and Applications in Tumor Therapy and in Food Industry. Microbiological Research 208: 99-112.

3. Wriston JC, TO Yellin (1973) L-Asparaginase: A Review. Advances in enzymology and related areas of molecular biology 39: 185-248.

ISSN: 2574-1241

DOI: 10.26717/BJSTR.2019.21.003584

Yago Queiroz dos Santos. Biomed J Sci \& Tech Res

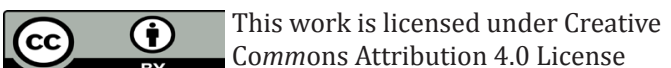

Submission Link: https://biomedres.us/submit-manuscript.php
4. Chen Shih Hsiang (2015) Asparaginase Therapy in Pediatric Acute Lymphoblastic Leukemia: A Focus on the Mode of Drug Resistance. Pediatrics \& Neonatology 56(5): 287-293.

5. Shrivastava, Abhinav, Khurshid M, Kalam MA, Jain SK et al. (2016) Recent Developments in L-Asparaginase Discovery and Its Potential as Anticancer Agent. Critical Reviews in Oncology/Hematology 100: 1-10.

6. Zuo Shaohua, Tao Zhang, Bo Jiang, Wanmeng Mu (2014) Recent Research Progress on Microbial L-Asparaginases. Applied Microbiology and Biotechnology 99(3): 1069-1079.

\section{Assets of Publishing with us}

BIOMEDICA RESEARCHES (+) $\mathrm{A}$ . 讨 . TP $\$$ ISSN: 2574-1241
- Global archiving of articles

- Immediate, unrestricted online access

- Rigorous Peer Review Process

- Authors Retain Copyrights

- Unique DOI for all articles 